\title{
Crossing the appositive / at-issue meaning boundary*
}

\author{
Scott AnderBois \\ UC Santa Cruz
}

\author{
Adrian Brasoveanu \\ UC Santa Cruz
}

\author{
Robert Henderson \\ UC Santa Cruz
}

\begin{abstract}
Our goal is to provide systematic evidence from anaphora, presupposition and ellipsis that appositive meaning and at-issue meaning, e.g. as contributed by the relative appositive and the main clause in John, who nearly killed a woman with his car, visited HER in the hospital, have to be integrated into a single, incrementally evolving semantic representation. While previous literature has provided partial arguments to this effect (Nouwen 2007 for anaphora, Amaral et al 2007 and Potts 2009 for both anaphora and presupposition), the systematic nature of this evidence - in particular, the evidence from ellipsis we will introduce - has been previously unnoticed. We propose an analysis of these phenomena that integrates the dynamic account of anaphora and ellipsis as discourse reference to individuals and properties (respectively) with an account of at-issue meaning as a proposed update of the input Context Set (CS) that is to be negotiated and of appositive meaning as an actual / imposed update of the CS that is not up for negotiation.
\end{abstract}

Keywords: appositives, dynamic semantics, propositional anaphora

\section{Introduction}

Since Potts 2005, it has been widely accepted that appositive content must be semantically separate from at-issue content. For example, (1), which has an appositive relative clause, seems to have a different interpretation from (2), where the same content is instead conjoined.

(1) John, who played tennis with a woman, played golf with her too.

(2) John played tennis with a woman and played golf with her too.

Potts (2005) formally captures this semantic separation through multidimensionality. This makes the strong prediction that the two meaning components will be wholly

* This research was supported by a Special Research Grant to Adrian Brasoveanu by the Committee on Research from the University of California, Santa Cruz. We want to thank Donka Farkas, Chris Potts, Floris Roelofsen, three anonymous SALT 20 reviewers, and the audience members of UCSC's S-Circle and SALT 20 for comments and discussion. 
separate with respect to all semantic phenomena. Recent literature has provided partial evidence against this strong separation, but its systematicity has been underappreciated. For example, Nouwen (2007) shows that at least certain kinds of anaphora cross the appositive / at-issue boundary freely and Amaral et al (2007) do so for both anaphora and presupposition; such facts were also noted by Potts (2005: 51-54) and Potts (2009). Drawing heavily on examples from Davies's (2008-) Corpus of Contemporary American English (COCA) we show that this pattern holds of anaphora generally, as well as most ellipsis processes.

We propose an analysis where appositive and at-issue content are part of the same meaning dimension, differing only in how they enter the Common Ground / Context Set (CG / CS, Stalnaker 1978). ${ }^{1}$ At-issue meaning is a proposal to update the input CS (a feature less emphasized in Stalnaker 1978 - for more discussion, see Farkas \& Bruce 2010 and references therein). In contrast, appositives are updates which are imposed on the CS and not up for negotiation by normal means.

Implementing this distinction, however, requires a semantics which processes both kinds of updates incrementally in order to properly capture 'boundary-crossing' phenomena like anaphora, ellipsis, and presupposition (see Amaral et al 2007 for more discussion of this point). For example, the pronoun HER in the main clause of (1) needs to find its antecedent within the appositive - and the presupposition of TOO must be satisfied by the propositional content of the appositive.

The paper is structured as follows. Section 2 shows the robust boundary-crossing behavior of a wide variety of anaphora, presupposition and ellipsis. Section 3 presents the account of apposition and boundary-crossing anaphora. Section 4 examines the behavior of clause-medial and clause-final appositive content in conversation more closely, and section 5 concludes.

\section{Phenomena crossing the boundary}

Based on data from anaphora and presupposition, Nouwen (2007) and Amaral et al (2007) have argued that appositive content cannot be wholly separate from at-issue content as multidimensionality would allow. In this section, we show that these arguments hold for cross-sentential anaphora and presuppositions quite systematically. Furthermore, we present novel data showing a parallel pattern for NP ellipsis and VP ellipsis. ${ }^{2}$ Finally, we show that all three of these processes operate freely in both directions, i.e. both at-issue $\rightarrow$ appositive and appositive $\rightarrow$ at-issue. The bi-directionality of boundary-crossing phenomena serves as crucial evidence in

1 We restrict our discussion to the simpler notion of CS for the remainder of the paper.

2 While ellipsis in general crosses the boundary freely, a systematic exception to this is sluicing, e.g., John, who once killed a man in cold blood, wondered who *(it was). See AnderBois 2010 for discussion and an account of this exception. 
favor of an incremental and interleaved account like the one in $\S 3$, in which the parts of the main and appositive clauses are interpreted left-to-right in the order in which they are uttered, as opposed to a sequential and non-interleaved account where full appositive content is somehow extracted and interpreted prior to at-issue content.

\subsection{Presupposition}

Presupposition with a variety of triggers is possible both in (a) the appositive $\rightarrow$ atissue direction and (b) the at-issue $\rightarrow$ appositive direction. This includes both strong and weak presupposition triggers in the sense of Abusch 2010: either in (3a) and (3b) below, too in (4a) and (4b), the restorative reading of again in (5a) and (5b), its non-restorative reading in (6) and, finally, the aspectual verb stop in (7a) and (7b).

(3) a. John, who wouldn't talk to Mary, wouldn't talk to SUSAN EITHER.

b. John wouldn't talk to Mary, who wouldn't talk to HIM EITHER.

(5) a. John, who has been sick, is now healthy AGAIN.

b. The window will be opened by Mary, who will then close it AGAIN.

(6) Suppliers produce a garment, double the cost and sell it to a retailer, who DOUBLES THE COST AGAIN and sells it to a consumer. (COCA)

(7) a. John, who is now building a sandcastle, will sTOP soon.

b. The sandcastle was only halfway built by John, who had suddenly STOPPED.

Finally, perhaps unsurprisingly, a presupposition introduced in one appositive can be cross-sententially retrieved in a subsequent appositive, as shown in (8) below.

(8) "Joe!" exclaims a young woman, who jumps in the air and throws her arms around him. "Joe! Joe! It's really you!" cheers a second young woman, who HUGS HIM TOO. (COCA)

\subsection{Anaphora}

Various types of anaphora exhibit the same kind of bi-directional boundary crossing behavior. Singular anaphora is exemplified in (9a) below, where the pronoun (her) can retrieve an antecedent in the appositive, and in (9b), where the pronoun and the presupposition retrieve antecedents in the main clause. Similarly, plural anaphora to (certain) quantifiers is felicitous, both in the at-issue $\rightarrow$ appositive direction (10a) and vice-versa (10b). Finally, modal anaphora and subordination is exemplified in (11a) and (11b) and quantificational subordination in (12a) and (12b). 
Crossing the appositive / at-issue meaning boundary

(9) a. John, who had been kissed by Mary, kissed HER TOO.

b. John kissed Mary, who kissed HIM TOO.

(10) a. Every speaker, all of THEM PhD students, gave a great talk.

b. Jones, who graded each student's final paper, gave THEM detailed feedback.

(11) a. John, who might give a presentation, WOULD use slides. Bill WOULD just use the board.

b. John might punch Jorge, who WOULD punch John back.

(12) a. Mary, who courts a semanticist at every conference party, ALWAYS dances with HIM.

b. Mary courts a semanticist at every conference party, where she ALWAYS dances with HIM.

\subsection{Ellipsis}

Data from NP- and VP-Ellipsis (NPE / VPE) point in the same direction. Since NPE arguably does not require a linguistic antecedent (i.e., is a type of deep anaphora in the sense of Hankamer \& Sag 1976), we might expect the examples in (13) and (14) to be possible regardless of the status of appositive content.

(13) a. Melinda, who won three games of tennis, lost because Betty won SIX.

b. Melinda lost three games of tennis to Betty, who lost SIX to Jane.

(14) a. The 1980's were dominated by the Lakers, who won five championships, and by the Boston Celtics, who won THREE. (COCA)

b. "When we've got four or five guys hitting threes," said guard Pat Bradley, who made THREE, ... (COCA)

But we find examples of VPE (a type of surface anaphora, requiring a linguistic antecedent) in both directions, as shown below.

(15) a. Mr. Gore at first believed the president, and even defended him to Tipper and his daughters, who DID NOT. (COCA)

b. So Lalonde, who was the one person who could deliver Trudeau, DID. (COCA)

As expected, we find the usual strict / sloppy ambiguity in (16a)-(16b) below - for example, (16a) can be interpreted as saying that Jane was told to help Mary's sister (strict reading) or Jane's (sloppy reading). This indicates that the appositive and at-issue components require access not only to each other's linguistic form, but also to their semantic representation. 
(16) a. Mary, who doesn't help her sister, told Jane TO.

b. John, who helps people if they want him to, kisses them even if they DON'T.

Finally, the example in (17) below exemplifies VPE from one appositive to another across at-issue items.

(17) I got a few quick words with Halle Berry, who looked amazing in Prada, and Sigourney Weaver, who DIDN'T. (COCA)

In sum, a wide variety of anaphora, presupposition, and ellipsis processes do not distinguish between appositive and at-issue content. These processes can operate in either direction, subject to linear order. We conclude (together with Amaral et al (2007) and Schlenker (2009)) that appositive and at-issue content is fundamentally unidimensional and incremental.

\section{The account}

The central challenge for an analysis of appositives is how to reconcile this robust unidimensionality with the data and intuition motivating Potts's (2005) multidimensional account. That is, how can we capture the contrast in (1-2), repeated as (18-19), in a unidimensional semantics?

(18) John, who played tennis with a woman, played golf with her too.

(19) John played tennis with a woman and played golf with her too.

The basic insight is that appositive and at-issue contents differ principally in how they enter the common ground. At-issue assertions are proposals to update the CG / CS (as argued in detail by Farkas \& Bruce (2010)), canonically subject to acceptance or rejection by other conversational participants. Appositive content, on the other hand, is imposed ${ }^{3}$ on the common ground, with little room for negotiation (responses to appositives will be discussed in detail in section 4).

This basic distinction can be phrased in quasi-Stalnakerian terms as follows. Let us take the designated propositional variable $p^{c s}$ to store the current CS. Then, the at-issue component puts forth a proposal, $p^{i s s u e}$, to update the CS by restricting possible future contexts to those that have non-empty intersections with $p^{i s s u e}$, namely $p^{c s} \cap p^{i s s u e}$. The addressee can accept or reject this proposal. If accepted, the $\mathrm{CS}$ is updated by assigning a new value to the variable $p^{c s}$, namely the intersection of the old $p^{c s}$ with $p^{i s s u e}$ :

3 Thanks to Floris Roelofsen for suggesting this term. 
Crossing the appositive / at-issue meaning boundary

$$
p^{c s}:=p^{c s} \cap p^{i s s u e}
$$

In contrast, an appositive imposes (as opposed to proposes) an update on the CS with its propositional content $p^{\text {appos}}$. In the terms of Farkas \& Bruce (2010), an appositive is not placed on the discourse table. Furthermore, in this simple version, appositive updates should always precede at-issue updates:

$$
p^{c s}:=p^{c s} \cap p^{a p p o s} ; p^{c s}:=p^{c s} \cap p^{i s s u e}
$$

Thus far, the approach is similar to Murray's (2009a; 2009b) approach to evidentials in Cheyenne. In these works, an evidential directly updates the common ground prior to the at-issue proposal (whether it is an assertion or a question).

\subsection{Two types of updates}

While this account in terms of sentence-level and sequential updates may work for evidentials, it fails to account for the phenomena discussed in section 2. Consider, for example, (22) below.

(22) John, who nearly killed a woman with his car, visited her in the hospital.

On the one hand, the content of the appositive cannot be determined independently of the at-issue component - the pronoun his in the appositive is anaphoric to the proper name John in the main clause. On the other hand, the content of the main clause cannot be determined independently of the appositive component - the pronoun her in the main clause is anaphoric to the indefinite $a$ woman in the appositive. Thus, we need to capture anaphora to properly determine propositional content. The appositive and at-issue updates need to be interwoven to resolve anaphora and presupposition, but we still need to distinguish at-issue proposals and appositive impositions.

As a first attempt, suppose we follow Heim (1982) and represent the CS by means of a designated world variable $w^{c s}$. At any point in discourse, the information state at that point consists of all the variable assignments that are still live options. The CS consists of the worlds assigned to the variable $w^{c s}$ by all these assignments and is encoded implicitly by the rows in (23):

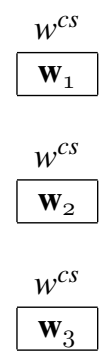


A sequence of (at-issue) updates is easy to capture - every update eliminates more and more assignments and, therefore, worlds associated with the variable $w^{c s}$.

(24) $\mathrm{John}^{x}$ nearly killed a ${ }^{y}$ woman with his $x$ car. He $x$ visited her $y$ in the hospital.

$$
\begin{array}{|l|}
w^{c s} \\
\hline \mathbf{w}_{1} \\
\hline
\end{array}
$$

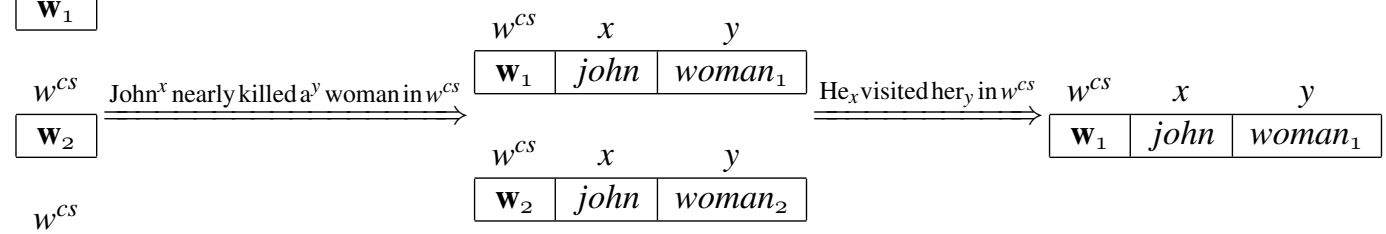

But if we model the CS by means of a world variable $w^{c s}$, there is only one way to update the CS: we eliminate worlds by eliminating assignments, incrementally restricting the CS. While both at-issue and appositive updates restrict the CS to one of its subsets, they do so in different ways, so we need two different ways to select subsets of the CS. We will accomplish this in three steps.

The first step is to model the CS by means of a propositional variable $p^{c s}$ that stores the current CS and all its non-empty subsets. For example, if the current CS is the set of worlds $\left\{\mathbf{w}_{1}, \mathbf{w}_{2}, \mathbf{w}_{3}\right\}$, the current information state, i.e., the set of assignments that are still live options in discourse, is as represented on the left-hand side below. For readability, we will graphically depict such information states as shown on the right-hand side.

$$
\begin{gathered}
p^{c s} \\
\left\{\mathbf{w}_{1}, \mathbf{w}_{2}, \mathbf{w}_{3}\right\} \\
\hline\left\{\mathbf{w}_{1}, \mathbf{w}_{2}\right\} \\
p^{c s} \\
\left\{\mathbf{w}_{1}, \mathbf{w}_{3}\right\} \\
p^{c s} \\
\left\{\mathbf{w}_{2}, \mathbf{w}_{3}\right\} \\
p^{c s} \\
\left\{\mathbf{w}_{1}\right\} \\
p^{c s} \\
\left\{\mathbf{w}_{2}\right\} \\
p^{c s} \\
\left\{\mathbf{w}_{3}\right\} \\
\hline
\end{gathered}
$$$$
\text { or simply: }
$$ 
Crossing the appositive / at-issue meaning boundary

The second step is taking appositives to contribute eliminative, Heim-style updates. For example, suppose that John nearly killed a woman with his car only in worlds $\mathbf{w}_{1}$ and $\mathbf{w}_{2}$. We will then eliminate all the assignments that assign to $p^{c s}$ at least one world in which this is not true. That is, we will eliminate all the assignments that assign to $p^{c s}$ a set that includes world $\mathbf{w}_{3}$.

(26) John, who nearly killed a woman with his car, visited her in the hospital.

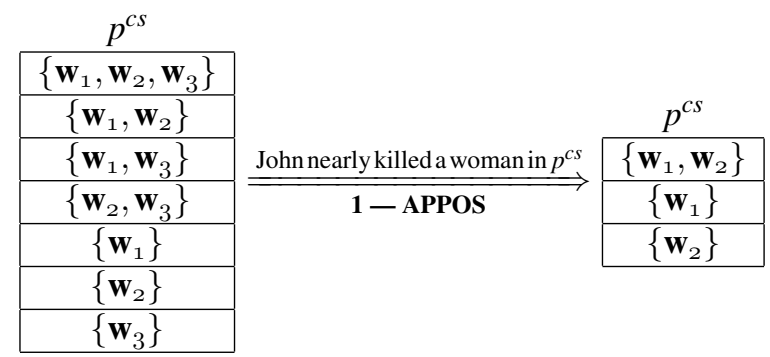

The appositive update is a test on the variable $p^{c s}$, just as woman $(y)$ is a test on the variable $y$, and contributes new information about the values of that variable.

The third and final step is to have at-issue updates put forth a proposal $p$ to update the CS by restricting the sets of worlds $p^{c s}$ to a subset $p \subseteq p^{c s}$. If the proposal is accepted, the new context set becomes $p$, provided that $p$ is non-empty. ${ }^{4}$ For example, suppose that John visited a woman in the hospital only in worlds $\mathbf{w}_{1}$ and $\mathbf{w}_{3}$. The proposal $p$ will store both these worlds or only one of them. If the proposal is accepted, the variable $p^{c s}$ is assigned the same values as $p$-and the power set previously stored in $p^{c s}$ is shrunk to a (much) smaller power set, as shown in (29).

(28) John visited a woman in the hospital.

\begin{tabular}{|c|c|c|c|}
\hline \multirow[b]{3}{*}{$p^{c s}$} & & \multicolumn{2}{|l|}{$p^{c s}$} \\
\hline & \multirow[b]{5}{*}{$p \subseteq p^{c s} \wedge$ John visited a woman in $p$} & $\left\{\mathbf{w}_{1}, \mathbf{w}_{2}, \mathbf{w}_{3}\right\}$ & $\left\{\mathbf{w}_{1}, \mathbf{w}_{3}\right\}$ \\
\hline & & $\left\{\mathbf{w}_{1}, \mathbf{w}_{2}, \mathbf{w}_{3}\right\}$ & $\left\{\mathbf{w}_{1}\right\}$ \\
\hline$\frac{p}{\left\{\mathbf{w}_{1}, \mathbf{w}_{2}, \mathbf{w}_{9}\right\}}$ & & $\left\{\mathbf{w}_{1}, \mathbf{w}_{2}, \mathbf{w}_{3}\right\}$ & $\left\{\mathbf{w}_{3}\right\}$ \\
\hline$\left\{\mathbf{w}_{1}, \mathbf{w}_{2}\right\}$ & & T $\left\{\mathbf{w}_{1}, \mathbf{w}_{2}\right\}$ & $\left\{\mathbf{w}_{1}\right\}$ \\
\hline$\left\{\mathbf{w}_{1}, \mathbf{w}_{3}\right\}$ & & $\left\{\mathbf{w}_{1}, \mathbf{w}_{3}\right\}$ & $\overline{\left\{\mathbf{w}_{1}, \mathbf{w}_{3}\right\}}$ \\
\hline$\left\{\mathbf{w}_{2}, \mathbf{w}_{3}\right\}$ & $\stackrel{1-\text { AT-ISSUE }}{\longrightarrow}$ & $\left\{\mathbf{w}_{1}, \mathbf{w}_{3}\right\}$ & $\left\{\mathbf{w}_{1}\right\}$ \\
\hline$\left\{\mathbf{w}_{1}\right\}$ & & $\left\{\mathbf{w}_{1}, \mathbf{w}_{3}\right\}$ & $\left\{\mathbf{w}_{3}\right\}$ \\
\hline$\left\{\mathbf{w}_{2}\right\}$ & & $\left\{\mathbf{w}_{2}, \mathbf{w}_{3}\right\}$ & $\left\{\mathbf{w}_{3}\right\}$ \\
\hline$\left\{\mathbf{w}_{3}\right\}$ & & $\left\{\mathbf{w}_{1}\right\}$ & $\left\{\mathbf{w}_{1}\right\}$ \\
\hline & & $\left\{\mathbf{w}_{2}\right\}$ & $\emptyset$ \\
\hline & & $\overline{\left.\mid \mathbf{w}_{3}\right\}}$ & $\left\{\mathbf{w}_{3}\right\}$ \\
\hline
\end{tabular}

4 In a technical sense, then, the output of the first update in (29) should also have one assignment which assigns $\emptyset$ to $p$ for each possible assignment of a value to $p^{c s}$. The 'provided that' clause in the third step ensures that such rows will be eliminated if $p$ is accepted, ruling out absurd updates. 


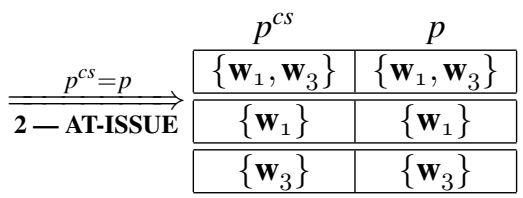

Thus, both at-issue and appositive updates contribute new information, i.e., restrict the values assigned to the variable $p^{c s}$. The appositive update is eliminative and targets the variable $p^{c s}$ directly. In contrast, the at-issue update contributes a proposal $p$ that is a subset of the CS variable $p^{c s}$. If the proposal is accepted, the set of worlds assigned to $p$ is also assigned to $p^{c s}$.

We can freely interleave these two ways of restricting the CS. That is, we make use of the fact that dynamic semantics keeps track of two kinds of interwoven information that can be updated simultaneously: (i) the factual, propositional information stored by the sets of worlds that are assigned as values to propositional variables $p, p^{\prime}, \ldots$, which is used to formalize the new information contributed by the at-issue update; (ii) the anaphoric information stored by variable assignments and incrementally constrained in discourse, which is used to formalize the new information the appositive update imposes.

Together, the two updates can be represented in the linear order in which they

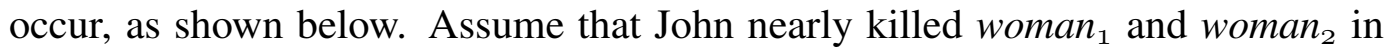
world $\mathbf{w}_{1}$ and, also, in world $\mathbf{w}_{2}$. Further assume that there is no near-killing in world $\mathbf{w}_{3}$. Finally, assume that John visited oman $_{1}$ in world $\mathbf{w}_{1}$ only.

$\mathrm{John}^{x}$, who $x$ nearly killed a ${ }^{y}$ woman with his $x$ car, visited her $y$ in the hospital.

\begin{tabular}{|c|c|c|c|c|}
\hline & & $p^{c s}$ & $p$ & $x$ \\
\hline & & $\left\{\mathbf{w}_{1}, \mathbf{w}_{2}, \mathbf{w}_{3}\right\}$ & $\left\{\mathbf{w}_{1}, \mathbf{w}_{2}, \mathbf{w}_{3}\right\}$ & john \\
\hline & & $\left\{\mathbf{w}_{1}, \mathbf{w}_{2}, \mathbf{w}_{3}\right\}$ & $\left\{\mathbf{w}_{1}, \mathbf{w}_{2}\right\}$ & john \\
\hline & & $\left\{\mathbf{w}_{1}, \mathbf{w}_{2}, \mathbf{w}_{3}\right\}$ & $\left\{\mathbf{w}_{1}, \mathbf{w}_{3}\right\}$ & john \\
\hline & & & $\cdots$ & \\
\hline$p^{c S}$ & & $\left\{\mathbf{w}_{1}, \mathbf{w}_{2}\right\}$ & $\left\{\mathbf{w}_{1}, \mathbf{w}_{2}\right\}$ & john \\
\hline$\left\{\mathbf{w}_{1}, \mathbf{w}_{2}, \mathbf{w}_{3}\right\}$ & & $\left\{\mathbf{w}_{1}, \mathbf{w}_{2}\right\}$ & $\left\{\mathbf{w}_{1}\right\}$ & john \\
\hline$\left\{\mathbf{w}_{1}, \mathbf{w}_{2}\right\}$ & & $\left\{\mathbf{w}_{1}, \mathbf{w}_{2}\right\}$ & $\left\{\mathbf{w}_{2}\right\}$ & john \\
\hline$\left\{\mathbf{w}_{1}, \mathbf{w}_{3}\right\}$ & $\stackrel{p \subseteq p^{c s} \wedge x=\mathrm{JOHN}}{\Longrightarrow}$ & $\overline{\left\langle\mathbf{w}_{1}, \mathbf{w}_{3}\right\}}$ & $\left\{\mathbf{w}_{1}, \mathbf{w}_{3}\right\}$ & john \\
\hline$\left\{\mathbf{w}_{2}, \mathbf{w}_{3}\right\}$ & 1 -AT-ISSUE & $\left\{\mathbf{w}_{1}, \mathbf{w}_{3}\right\}$ & $\left\{\mathbf{w}_{1}\right\}$ & john \\
\hline$\left\{w_{1}\right\}$ & & $\left\{\mathbf{w}_{1}, \mathbf{w}_{3}\right\}$ & $\left\{\mathbf{w}_{3}\right\}$ & john \\
\hline $\begin{array}{l}\left\{\mathbf{w}_{2}\right\} \\
\left\{\mathbf{w}_{1}\right\}\end{array}$ & & 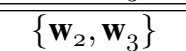 & $\left\langle\mathbf{w}_{2}, \mathbf{w}_{3}\right\}$ & john \\
\hline$x_{3} \mathrm{~s}$ & & $\left\{\mathbf{w}_{2}, \mathbf{w}_{3}\right\}$ & $\left\{\mathbf{w}_{2}\right\}$ & john \\
\hline & & $\left\{\mathbf{w}_{2}, \mathbf{w}_{3}\right\}$ & $\left\{\mathbf{w}_{3}\right\}$ & john \\
\hline & & $\left\{\mathbf{w}_{1}\right\}$ & $\left\{\mathbf{w}_{1}\right\}$ & john \\
\hline & & $\left\{\mathbf{w}_{2}\right\}$ & $\left\{\mathbf{w}_{2}\right\}$ & john \\
\hline & & $\left\{\mathbf{w}_{3}\right\}$ & $\left\{\mathbf{w}_{3}\right\}$ & john \\
\hline
\end{tabular}


Crossing the appositive / at-issue meaning boundary

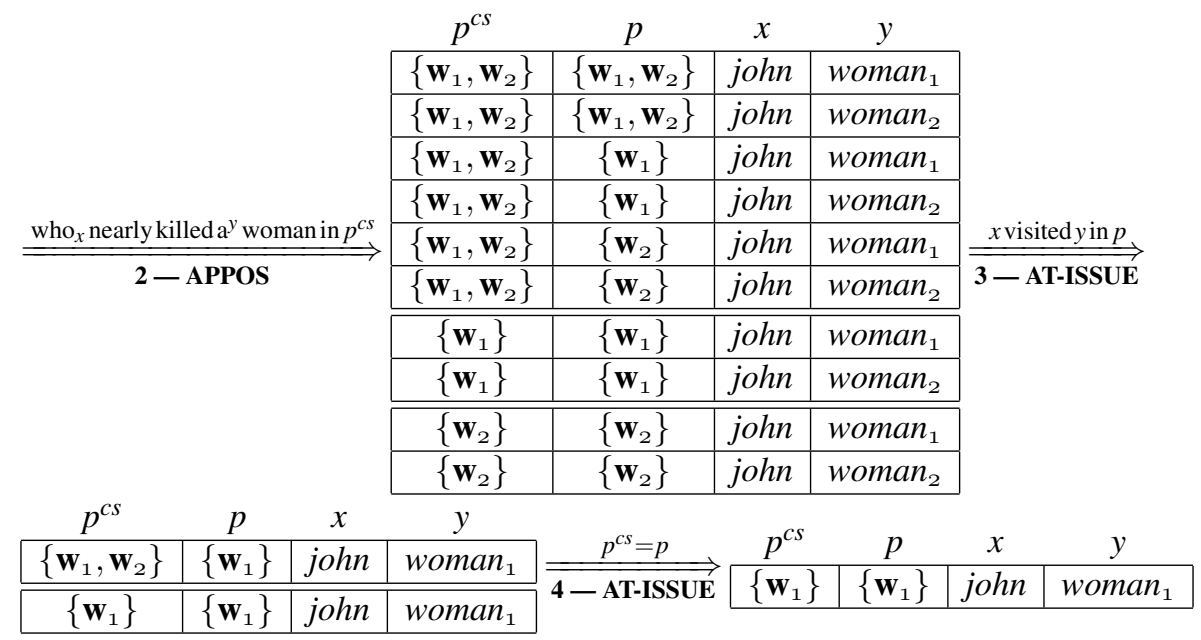

\subsection{Appositives vs presuppositions}

Like appositives, a presupposition targets the input CS through the dref $p^{c s}$ directly. Neither presuppositions nor appositives are part of the at-issue proposal $p$ to update the CS. However, there is a fundamental difference between them. Aside from their non-negotiability, appositives are ordinary updates of the current information state (i.e., the current CS in Stalnakerian terms). A felicitous use of an appositive, then, is truth-conditionally informative.

In contrast, presuppositions are constraints or preconditions on the current information state / CS. They are required to be satisfied throughout the entire input information state / CS. That is, any assignment $g$ in the input information state has to satisfy the presupposition..$^{5}$ Presupposed meaning is taken for granted and anaphorically links the non-presupposed (at-issue and / or appositive) meaning with its context of interpretation. Presuppositions which are not met throughout the input CS can, of course, be accommodated. Such accommodation, however, is an exceptional means of repairing the input information state / CS, rather than being the general case. Thus, we take anaphoricity to be an important part of what it means to presuppose something, following van der Sandt (1992) and others (see Kamp 2001 among others for a more recent discussion).

Given the characterization of presuppositions as preconditions on the CS as opposed to appositives, which are tests - we correctly expect that appositives themselves may contribute presuppositions. For example, the appositive update of (32) presupposes that John has a Ferrari and that he washed it prior to today.

(32) John, who washed his Ferrari again today, is completely obsessive.

5 We can formalize this along the lines of van der Sandt 1992; see also the discussion in Krahmer 1998: Ch. 6. 
Finally, note that presuppositions in an appositive can be satisfied by at-issue content, as in (9b) above (John kissed Mary, who kissed HIM TOO). This occurs with presuppositions that are hard to accommodate (too), so we predict that the appositive forces the acceptance of the at-issue proposal prior to the appositive update. Thus, proposals to update the common ground do not come only in sentence/clause-sized chunks. This is very much in line with Clark \& Schaefer (1989) and others, who observe that discourse negotiation mostly takes place at sub-clausal level.

\subsection{Dynamic proposals}

Formally, we can produce these two types of updates in an extension of Dynamic Predicate Logic (Groenendijk \& Stokhof 1991).

Models consist of the disjoint domains of individuals $\mathfrak{D}$ and possible worlds $\mathfrak{W}$ and the basic interpretation function $\mathfrak{I}$, which assigns a subset of $\mathfrak{D}^{n}$ to any $n$-ary relation $R$ relative to any world $w: \mathfrak{I}_{w}(R) \subseteq \mathfrak{D}^{n}$. We have variables over individuals $(x, y, \ldots)$, worlds $\left(w, w^{\prime}, \ldots\right)$ and propositions / sets of worlds $\left(p, p^{\prime}, p^{c s}, \ldots\right)$ and the

usual inventory of non-logical constants: individual constants $(\mathrm{JOHN}, \ldots)$, properties (WOMAN,$\ldots$ ), binary relations (VISIT,$\ldots$ ) etc.

Formulas are interpreted relative to a pair of assignments $\langle g, h\rangle$, i.e., they denote binary relations between an input assignment $g$ and an output assignment $h$. In particular, dynamic conjunction is interpreted as relation composition:

$$
[[\phi \wedge \psi]]^{\langle g, h\rangle}=\mathbb{T} \text { iff there exists a } k \text { such that }[[\phi]]^{\langle g, k\rangle}=\mathbb{T} \text { and }[[\psi]]^{\langle k, h\rangle}=\mathbb{T}
$$

New variables are introduced by means of random assignment formulas $[x],[p]$, etc.

$[[[v]]]^{\langle g, h\rangle}=\mathbb{T}$ (for any variable $v$ ) iff $g$ differs from $h$ at most with respect to the value assigned to $v-$ i.e., for any variable $v^{\prime} \neq v, g\left(v^{\prime}\right)=h\left(v^{\prime}\right)$

Lexical relations relativized to propositions are distributively interpreted.

$$
\left[\left[R_{p^{c s}}(y)\right]\right]^{\langle g, h\rangle}=\mathbb{T} \text { iff } g=h \text { and for all worlds } w \in h\left(p^{c s}\right), h(y) \in \mathfrak{I}_{w}(R)
$$

\subsection{Discourse reference across the boundary}

Sentence (36) (repeated from above) is represented as in (37) below.

(36) John ${ }^{x}$, who nearly killed a ${ }^{y}$ woman with his $x$ car, visited her $y$ in the hospital.

(37) a. New proposal: $[p] \wedge p \subseteq p^{c s} \wedge$

b. Issue: $[x] \wedge x=\mathrm{JOHN} \wedge$

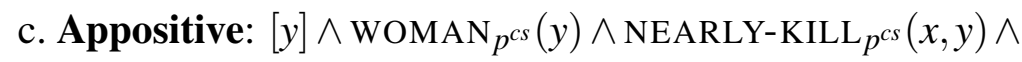


Crossing the appositive / at-issue meaning boundary

d. Issue: $\operatorname{VISIT}_{p}(x, y) \wedge$

e. Proposal accepted: $\left[p^{c s}\right] \wedge p^{c s}=p$

The formula in (37a) introduces the proposal to update the CS: we introduce a new variable $p \subseteq p^{c s}$ containing worlds satisfying the subsequent at-issue update. The formulas in (37b) and (37d) are the two at-issue updates and the formula in (37c) is the appositive update. They instruct us to introduce a new variable $x$ whose value is John and comment that $x$ nearly killed a woman $y$ and $x$ visited $y$.

The appositive nature of the update in (37c) is captured by the fact that the appositive content is interpreted relative to $p^{c s}$ rather than relative to the new proposal $p$. The following update in $(37 \mathrm{~d})$, i.e., $\operatorname{VISIT}_{p}(x, y)$, is part of the at-issue proposal, so it is interpreted relative to $p$.

More needs to be said about how this subscripting arises compositionally. The most straightforward way to implement this is by using left and right comma operators following Nouwen (2007). While for Nouwen, these operators toggle back and forth between two Pottsian dimensions, for us, they toggle back and forth between $p^{c s}$ and $p$. That is, a left comma operator indicates that subsequent content should update $p^{c s}$ while the right comma toggles back to updating $p$ itself. Since the left comma operator picks out $p^{c s}$ directly, this accounts for the fact that appositives, even inside of attitude reports, are speaker-oriented. Following arguments in Harris \& Potts 2009, we take cases of non-speaker orientation to be the result of pragmatically-driven perspective shift, separate from the semantics of appositives. For a dynamic account of related cases of modal anaphora, see Brasoveanu 2010.

Finally, (37e) contributes the proposal to update the CS variable $p^{c s}$ by resetting it to $p$. Despite the possible non-maximality of the set of worlds $p$, the Stalnakerian CS (which is the maximal set of worlds compatible with both the previous CS and the at-issue proposal) will always be recoverable: after the update in (37d), there will be an output assignment $h$ such that $h(p)$ contains the maximal set of worlds in the current CS that satisfy the at-issue relation $\operatorname{VISIT}_{p}(x, y)$. Therefore, the output information state will necessarily have an output assignment storing this maximal set of worlds, which is the new CS in Stalnaker's sense. ${ }^{6}$

Since the account is fundamentally unidimensional, ellipsis processes like VPE can be accounted for straightforwardly. One way to accomplish this is to extend the dynamic framework with discourse referents / variables for properties along the lines of Hardt 1999 and Stone \& Hardt 1999. Just as the indefinite $a$ woman in the appositive in (36) introduces a discourse referent, the antecedent VP does too. Retrieving the VP in the ellipsis site is similarly parallel to the way in which

6 In other words: let $H$ be the set of output variable assignments obtained after the sequence of updates in (37) above. The new CS in Stalnaker's sense is the maximal set of worlds in the set $\left\{h\left(p^{c s}\right): h \in H\right\}$ or, alternatively, the set of worlds $\bigcup_{h \in H} h\left(p^{c s}\right)$. 
the pronoun her in the main clause anaphorically retrieves the discourse referent introduced by the indefinite.

\section{Appositives in discourse}

The account proposed in $\S 3$ treats appositives as updates which are imposed on the common ground. In this section, we explore the ways in which appositive content behaves in discourse, showing how they follow from this characterization.

\subsection{Proposals and questions under discussion}

One central difference between at-issue and appositive content is the relative inability of the latter to interact with the question under discussion (QUD). ${ }^{7}$ While apparent examples of appositive questions occur in written English, as in (38-39), they are quite infelicitous in spoken English. That is, appositive content cannot explicitly introduce a new QUD the way that at-issue content can.

(38) This unknown person fell in love with Carlos, and, in a moment of rage and jealousy (who knew what Carlos felt?) beat Warren to death. (COCA)

(39) The producers are on vacation in Hawaii. Larry (who's Larry?) is on the golf course and can't be reached. (COCA)

Parallel to this, appositive content cannot readily resolve an existing QUD. We see this plainly in the contrast (40) vs (41): while the appositive content in (40) clearly resolves the question, it's appositive nature makes it an infelicitous answer.

a. Who had prostate cancer?

b. ??Tammy's husband, who had prostate cancer, was being treated at the Dominican Hospital.

(41) a. Who was being treated at the Dominican Hospital?

b. Tammy's husband, who had prostate cancer, was being treated at the Dominican Hospital.

These facts follow directly from our characterization of appositive content as not being placed on the table, in the terms of Farkas \& Bruce (2010). The table is not only where the acceptance / rejection of assertions take place, it is also where the QUD stack is managed. One central claim of Farkas \& Bruce (2010) is that serving as a proposal to update the CS intrinsically involves the same discourse resources

7 For the notion of question under discussion, see Roberts 1996, Ginzburg 1996, Büring 2003 and Farkas \& Bruce 2010 among others. 
Crossing the appositive / at-issue meaning boundary

as managing the QUD. Farkas \& Bruce (2010) support this claim empirically by examining the parallels between responses to at-issue assertions and polar questions. Since the structure of the table is what relates at-issue content to the QUD, it follows that content which imposes an update on the CS itself (as we claim for appositives) cannot interact with the QUD.

\subsection{Responding to appositive content}

Another contrast between appositives and at-issue assertions is the range of possible responses an addressee can give to them. Farkas \& Bruce (2010) show that at-issue assertions allow for roughly the same range of responses that polar questions expect. In particular, assertions readily allow for bare particle responses like yes, no, and maybe as in (42) below.

(42) a. A: Sonia is coming to the party.

b. B: Yes // No // Maybe // Perhaps.

In contrast, bare particle responses are not readily interpreted as ratifying appositive content, as the contrast in continuations between (43) and (44) below show.

(43) a. A: Sonia, who is a terrible housemate, left the door unlocked last night.

b. B: Yeah, but she is still a good housemate.

c. B: No, but she is a terrible housemate.

(44) a. A: Sonia is a terrible housemate and she left the door unlocked last night.

b. B: \#Yeah, but she is still a good housemate.

c. B: \#No, but she is a terrible housemate.

While bare particle responses do not readily target appositive content, responses that echo or expand on the appositive content are possible:

COCA, 60 Minutes, CBS Sixty.

a. [Mr. DON FUQUA] He told me about Noah, his first-born, and how he shared his son's love of rockets. He told me about how thankful he was to have Mary, his only girl, and Luke, who loved to have his picture taken.

b. [SPOKESMAN] Yeah, he always liked the camera. He'd always smile, but he always squint his eyes and ...

COCA, Lisa Ling goes inside one of the world's most dangerous gangs; journalists Lisa Ling, Anderson Cooper and Brian Ross discuss some of their most memorable stories, Ind Oprah. 
a. [Mr. ANDERSEN] And there was some sense of justice, I think, for these children for me to track down this foster mother, who really got away with outrageous behavior.

b. [Ms. SALTZMAN] Yeah. She got away with it.

Denying appositives is less frequent, but also possible. A COCA search for ", who" followed by "no" in a 9-word window, revealed no clear example of appositive denial, but discourses like the following seem to be nonetheless natural:

(47) a. He took care of his husband, who had prostate cancer.

b. No, he had lung cancer.

c. No, he took care of his brother.

(48) a. He told her about Luke, who loved to have his picture taken.

b. No, he didn't like that at all.

c. No, he told her about Noah.

However, both of these sorts of responses rely crucially on the utterance-final nature of the appositives in question. For example, parallel examples to (47-48), below in (49-50) sound quite degraded.

(49) a. His husband, who had prostate cancer, was being treated at the Dominican Hospital.

b. ??No, he had lung cancer.

c. No, he was being treated at the Stanford Hospital.

(50) a. Luke, who loved to have his picture taken, was his son.

b. ??No, he didn't like that at all.

c. No, Luke was his nephew.

Finally, in cases where the appositive is utterance-final, a speaker can require explicit confirmation through the use of tags such as right:

COCA, Tammy Faye Messner discusses the rise and fall of the PTL, CNN King.

a. $[\mathrm{Mr}$. HAHN] And take care of your husband, who has prostate cancer, right?

b. [WILLIAM DALEY] Yes.

c. $[\mathrm{Mr} . \mathrm{HAHN}]$ How's he doing?

(52) COCA, Charles Bryan earns right to play with Will Shortz, NPR Weekend. 
Crossing the appositive / at-issue meaning boundary

a. [ANNOUNCER] Happy Mother's Day to your mother today, who I guess is in town with you, right?

b. [ANNOUNCER] That's right, I flew her to New York as a Mother's Day gift.

Note also that, in both of these cases, right can be felicitously replaced with an opposite polarity tag question - doesn't he? and isn't she?, respectively.

\subsection{Medial vs final appositives}

We have seen that yes/no responses and the possibility of tags like right exhibit an asymmetry between appositives that are clause-medial (or perhaps utterance-medial) and those that are clause-final. In this section, we present data from presupposition satisfaction and the temporal adverb then which further cement the divide between medial and final appositives. The empirical generalization we arrive at is that final appositives enjoy a broader range of possible interpretations, behaving in many respects as though they were conjunctions ${ }^{8}$ rather than true appositives.

In addition to the data involving responses and tags above, the presupposition facts discussed at the end of $\S 3.2$ also seem to exhibit an asymmetry between medial and final appositives. While presuppositions in clause-final appositives can be easily satisfied by at-issue material, parallel examples with clause-medial appositives are quite difficult to construct:

(53) John kissed Mary, who kissed HIM TOO.

(54) ??John kissed Mary, who kissed HIM TOO, at the concert in the park.

A further set of facts that demonstrate an asymmetry between medial and final appositives is the interpretation of the temporal adverb then. In clause-final appositives like (55), then most readily indicates that the event in the appositive took place at a later time than the event in the main clause (a rough paraphrase being subsequently). In clause-medial appositives like (56), however, then indicates that the event described in the appositive took place at the same time as the at-issue event, a rough paraphrase being at that time.

(55) Every springtime they migrate out of the sea and swim upriver to reproduce by giving birth to live young, who then spend an undetermined amount of time living on land as what we call zucchini slugs. (COCA)

(56) In fact, while she was filming Fresh Prince, her mother, who then worked as a librarian, would call her with Black history stories. (COCA)

8 Thanks to Tamina Stephenson for this characterization. 
Taken together, these observations demonstrate that despite having the same surface form (at least in English), there is a persistent asymmetry between clause-final and clause-medial appositives — an idea previously suggested by del Gobbo (2003), based on quite different data (the relative felicity of clause-final appositives anchored on quantifiers like many and most). While it's not exactly clear how del Gobbo's (2003) data fit into this picture, the data from responses, tags, presupposition, and then all seem to suggest that final appositives often behave more like conjunctions or separate sentences rather than true appositives. ${ }^{9}$

\section{Conclusion}

To summarize, the robust patterns of anaphora, ellipsis and presupposition between at-issue and appositive meaning demand a unidimensional account. Our proposal to capture this unidimensionality while maintaining the distinction between at-issue and appositive meaning makes crucial use of the fact that dynamic semantics keeps track of two kinds of interwoven information that can be updated simultaneously: the factual, propositional information stored by propositional variables $p, p^{\prime}, \ldots$, which we use to formalize at-issue updates, and the anaphoric information stored by variable assignments, which we use to formalize the new information contributed by appositive updates. The account, then, makes use of independently motivated tools to distinguish between at-issue and appositive content, averting the need for dimensions as an additional theoretical construct above and beyond what is already motivated by at-issue content alone. Our richer representation of the CS as a set of sets of worlds plays a crucial role in distinguishing these two update types.

\section{References}

Abusch, Dorit. 2010. Presupposition triggering from alternatives. Journal of Semantics 27(1). 37-80. doi:10.1093/jos/ffp009.

Amaral, Patricia, Craige Roberts \& E. Allyn Smith. 2007. Review of "The Logic of Conventional Implicatures". Linguistics and Philosophy 30(6). 707-749. doi:10.1007/s10988-008-9025-2.

AnderBois, Scott. 2010. Sluicing as anaphora to issues. (Proceedings of SALT 20).

9 While we leave the analysis of this asymmetry to future work, we would like to highlight one possible direction for such an account, suggested to us by Nicholas Asher. The idea is that final appositives have a wider range of interpretations because they can enter into matrix-level discourse relations in a discourse structure (e.g. Asher \& Lascarides 2003). Medial appositives would be subject to more constraints on their interpretation because they would be discourse-subordinate to the clause they are syntactically attached to, while final appositives need not be. 
Crossing the appositive / at-issue meaning boundary

Asher, Nicholas \& Lascarides, Alex. 2003. Logics of conversation. Cambridge University Press.

Brasoveanu, Adrian 2010. Decomposing modal quantification. Journal of Semantics 27(4). 437-527. doi:10.1093/jos/ffq008.

Büring, Daniel. 2003. On D-trees, Beans and B-accents. Linguistics and Philosophy 26(5). 511-545. doi:10.1023/A:1025887707652.

Clark, Herbert \& Edward Schaefer. 1989. Contributing to discourse. Cognitive Science 13(2). 259-294. doi:10.1207/s15516709cog1302_7.

Davies, Mark (2008-). The Corpus of Contemporary American English (COCA): 400+ million words, 1990-present. Available online at http://www. americancorpus.org.

Farkas, Donka \& Kim Bruce. 2010. On reacting to assertions and polar questions. Journal of Semantics 27(1). 81-118. doi:10.1093/jos/ffp010.

Ginzburg, Jonathan 1996. Dynamics and the semantics of dialogue. Language, Logic and Computation. Vol. 1, Jerry Seligman \& Dag Westerståhl (eds.), CSLI, 221-237.

del Gobbo, Francesca. 2003. Appositives at the Interface. PhD Thesis, Univ. of California, Irvine.

Groenendijk, Jeroen \& Martin Stokhof. 1991. Dynamic predicate logic. Linguistics and Philosophy 14(1). 39-100. doi:10.1007/BF00628304.

Hankamer, Jorge \& Ivan Sag. 1976. Deep and surface anaphora. Linguistic Inquiry 7(3). 391-428. http://www.jstor.org/stable/4177933

Hardt, Daniel. 1999. Dynamic interpretation of Verb Phrase Ellipsis. Linguistics and Philosophy 22(2). 187-221. doi:10.1023/A:1005427813846.

Harris, Jesse \& Chris Potts. 2009. Perspective-shifting with appositives and expressives. Linguistics and Philosophy 32(6). 523-552. doi:10.1007/s10988-0109070-5.

Heim, Irene. 1982. The semantics of definite and indefinite noun phrases, $\mathrm{PhD}$ dissertation, University of Massachusetts, Amherst, published in 1988 by Garland, New York.

Kamp, Hans. 2001. The importance of presupposition. Linguistic Form and its Computation. Selected papers from the SFB 340. Christian Rohrer, Antje Roßdeutscher \& Hans Kamp (eds.), CSLI. 207-254.

Krahmer, Emiel. 1998. Presupposition and anaphora. CSLI.

Murray, Sarah. 2009a. A Hamblin semantics for evidentials. Proceedings of SALT $X I X$.

Murray, Sarah. 2009b. Evidentials and questions in Cheyenne. Proceedings of SULA 5: Semantics of Under-represented Languages in the Americas.

Nouwen, Rick. 2007. On appositives and dynamic binding, Research on Language and Computation 5(1). 87-102. doi:10.1007/s11168-006-9019-6. 
Potts, Christopher. 2005. The Logic of Conventional Implicatures. Oxford University Press.

Potts, Christopher. 2009. The dynamics of apposition. Handout from invited talk at Chris Barker's NYU Linguistics seminar on dynamics, February 25, 2008.

Roberts, Craige. 1996. Information Structure: Towards an integrated theory of formal pragmatics. OSU Working Papers in Linguistics 48. Jae-Hak Yoon \& Andreas Kathol (eds.), 91-136.

van der Sandt, Rob. 1992. Presupposition Projection as anaphora resolution. Journal of Semantics 9(4). 333-377. doi:10.1093/jos/9.4.333.

Schlenker, Philippe. 2009. Supplements within a Unidimensional Semantics I: Scope. To appear in the proceedings of the Amsterdam Colloquium 2009. Maria Aloni $\&$ Katrin Schulz (eds).

Schlenker, Philippe. 2009. Supplements within a Unidimensional Semantics II: Epistemic status and projection. To appear in the proceedings of North East Linguistic Society 2009.

Stalnaker, Robert. 1978. Assertion. Syntax and Semantics 9. 315-332.

Stone, Matthew \& Daniel Hardt. 1999. Dynamic discourse referents for tense and modals. Proceedings of the Second International Workshop on Computational Semantics. 287-299.

Scott AnderBois

UCSC, Linguistics

1156 High St, Santa Cruz

CA 95064

shanders@ucsc.edu

Robert Henderson

UCSC, Linguistics

1156 High St, Santa Cruz

CA 95064

rmh@ucsc.edu
Adrian Brasoveanu

UCSC, Linguistics

1156 High St, Santa Cruz

CA 95064

abrsvn@ucsc.edu 